\title{
Modelling and predicting asphalt deflection values with artificial neural networks
}

\author{
M. Rahimi Nahoujy \\ Ruhr-Universität Bochum, Bochum, Germany \\ The Federal Highway Research Institute, Bergisch Gladbach, Germany \\ M. Radenberg \\ Ruhr-Universität Bochum, Bochum, Germany
}

\begin{abstract}
The falling weight deflectometer (FWD) is an internationally used device for measuring deflections and evaluating the quality of pavement. But FWD can only take measurements of a limited number of kilometers of road per day. On a large scale, this method is expensive in terms of time and resources. Moreover, in some cases, data sets obtained from FWD are not sufficient, or they may be limited or missing, which makes the planning of maintenance and rehabilitation measures more error-prone. Aiming to overcome these limitations and to improve the method, in this work a new artificial neural network (ANN) approach was developed in order to calculate the deflection at any arbitrary point on the entire route to complement and substitute experimental measurements. A feed-forward ANN model was developed in the MATLAB computing environment based on backpropagation by a multilayer perceptron (MLP) network for asphalt pavement. A MLP neural network model was chosen, because it can deal with those cases, in which FWD deflection data is not sufficiently available. These networks are particularly suited to modeling complex data, as provided by FWD, due to the capability of ANN to learn complex nonlinear behavior. With at least 150 data sets, a model can be trained through ANN, that has a mean square error of less than 1 percent. Fewer measuring points are needed because the missing data can be calculated from the formulation. Thus, this method offers a great potential for the optimization of the traditional measurements in two ways: First, it optimizes measurement costs, and second, it significantly enhances the accuracy of road maintenance planning. Besides, it helps solving the problem of limited or missing data sets.
\end{abstract}

Keywords: Artificial neural network, multilayer perceptron, falling weight deflectometer, non-destructive testing, deflection

\section{INTRODUCTION}

Road maintenance costs are a considerable factor in managing public budgets, which means that finding efficient methods for the maintenance of these roads is of utmost importance. For economically optimized, technically adapted and demand-oriented planning, regular evaluations at appointed times are fundamental in every method of pavement maintenance.

Non-Destructive Tests (NDTs) are employed at different levels, to evaluate, repair, maintain, and rehabilitate different pavements. For optimal maintenance planning, especially for rehabilitation measures based on technical and economic criteria, it is important to consider the results of bearing capacity measurements (Jansen 2009). Therefore, the deflection basin 
determined in Non-Destructive Deflection Testing is commonly used to evaluate the structural conditions of surface pavement. Deflection measurements at defined load pulses with a falling weight deflectometer (FWD) are a method for load capacity measurements that has been used worldwide since the 1960s (Jansen 2009).

FWD is a global and advanced method that measures load capacities and deflection changes in asphalt pavement under load. The physical properties of asphalt pavement can be measured by this device. FWD data is mainly used to estimate the capacity of layers of asphalt pavement. In other words, it is used to determine if a road surface is overloaded or not.

Even though FWD is a popular device for the planning of maintenance and rehabilitation measures of roads and widely used in Europe, the US and beyond, the technique has a few considerable downsides. The FWD can only take measurements of a limited number of kilometers of road per day. On a large scale, this method is expensive in terms of time and resources. Moreover, in some cases, data sets obtained from FWD are not sufficient, or they may be limited or missing, which makes the planning of maintenance and rehabilitation measures more error-prone.

Nowadays, an artificial neural network (ANN) is a very effective tool in the domain of pavement maintenance (Nejad and Zakeri 2012, Attoh-Okine 1999). A number of studies has demonstrated that ANNs can be used for prediction in the domain of pavements maintenance. By modelling FWD data, the deflection can be predicted at any arbitrary point on the entire route measured by FWD.

The successful use of ANNs in pavements has been demonstrated in many studies, most of them focused on the backcalculation of the modulus for each pavement layer considering complex material properties in viscoelastic and nonlinear modulus (Kim and Kim 1998, Ceylan 2005, Ceylan et al. 2007, Li and Wang 2019;). Some works also document the success of ANN in directly (without backcalculation) predicting pavement responses (Saltan and Terzi 2007, Wang et al. 2020, Rahimi Nahoujy 2020).

\section{FWD TESTING DEVICE}

The FWD Primax (Figure 1) is one of the earliest deflectometers and has been commercially available since 1969 in Europe and the United States. In this device, an impact load of 10 to $50 \mathrm{kN}$ is generated by a weight released from various heights. The load is transmitted to the road surface (pavement) via a plate $(300 \mathrm{~mm}$ diameter). The deflections are measured by ten transducers, one of which is located at the center of the plate, while the rest are located at a distance of $2100 \mathrm{~mm}$ from the plate center (Huang 2004).

An operator can perform around 50 measurements per hour using a standard version of the Primax 1500 in Germany. The distance between points is 25 meters, and the applied load is $50 \mathrm{kN}$. The time history of the impulse load is recorded via a load cell. The impulse load generated by the FWD is used to simulate a wheel overrun and, therefore, should have the same pulse duration as the one resulting from a wheel overrun with a vehicle (pulse duration between 20 and $30 \mathrm{~ms}$ ). The size of the impulse load should correspond to the wheel load of a truck (Jansen 2009, FGSV 2008).

Geophones record the short-term vertical deformation of the road surface (deflection bowl) in response to the impulse load (FGSV 2008). In this study, one geophone is located in the load center, and the other nine are located at following distances from the load center: 0-200-300-450-600-900-1.200-1.500-1.800 (mm). In addition to FWD measurements, the temperature is measured at any point and the asphalt thickness is measured at all points by the ground penetrating radar (GPR) device. 


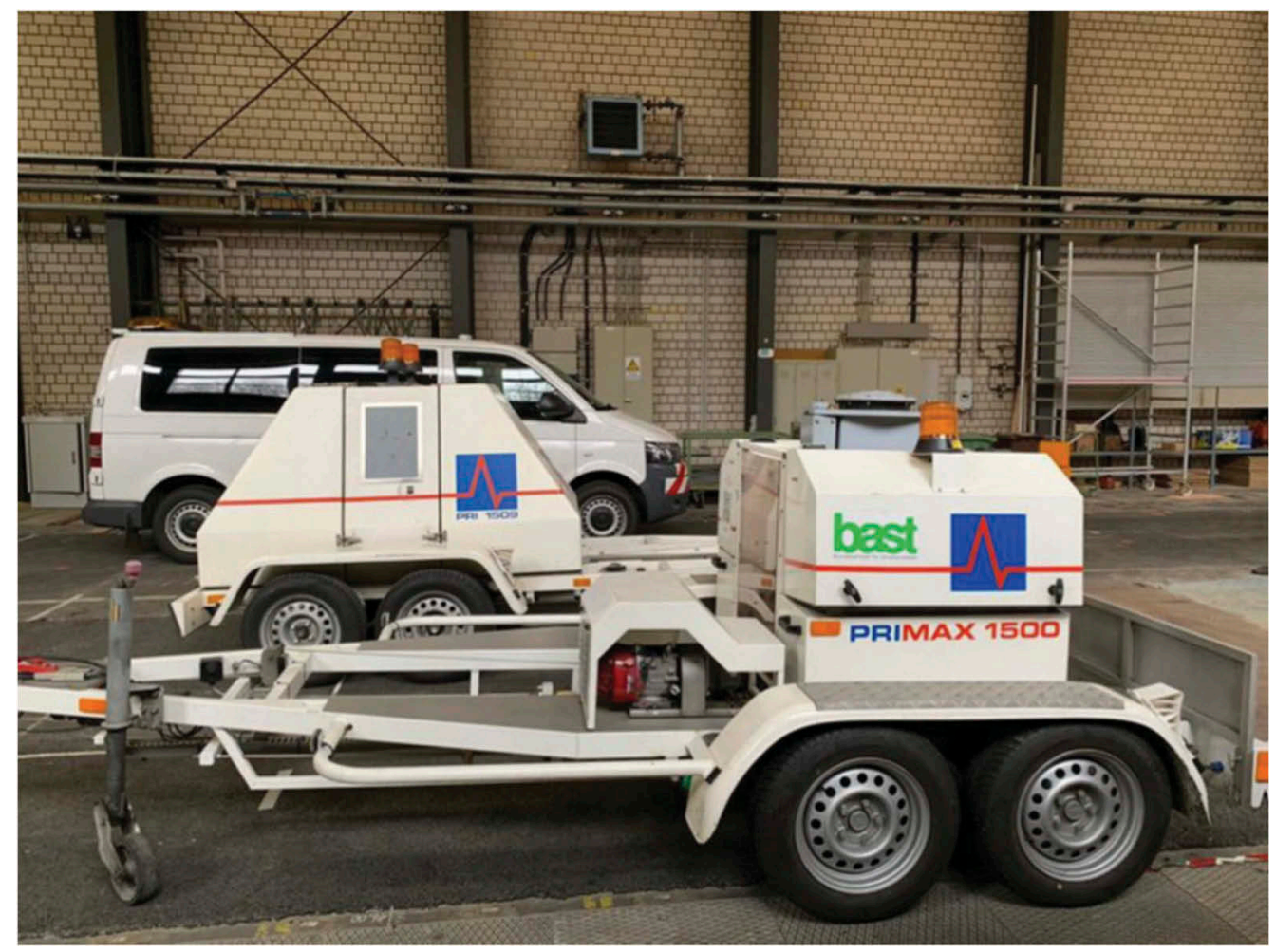

Figure 1. Primax 1500 FWD.

\section{METHODOLOGY}

\section{$3.1 A N N$}

ANNs are introduced as a useful tool in solving engineering problems with highly nonlinear functional approximations. ANNs have been successfully applied to tasks such as recognition of function approximation, function optimization, prediction, data recovery, automatic control and many other cases.

An ANN consists of a group of artificial neurons, which are the smallest unit of information processing. Every artificial neuron receives inputs, processes them, and finally produces an output signal. Two or more neurons can be combined in the form of a layer; an individual network can be made up of multiple layers. Each layer in the weight matrices network may have its biased vector and output.

An ANN is created with a small group of artificial neurons, receptive to training, aiming at solving complex problems. The grouping of artificial neurons can be described as mapping training, which means the neurons learn to create a mapping between the explanatory variables and the response variables (Hagan et al. 2014). The network displayed in Figure 2 includes input neurons, output neurons, and hidden neurons. 


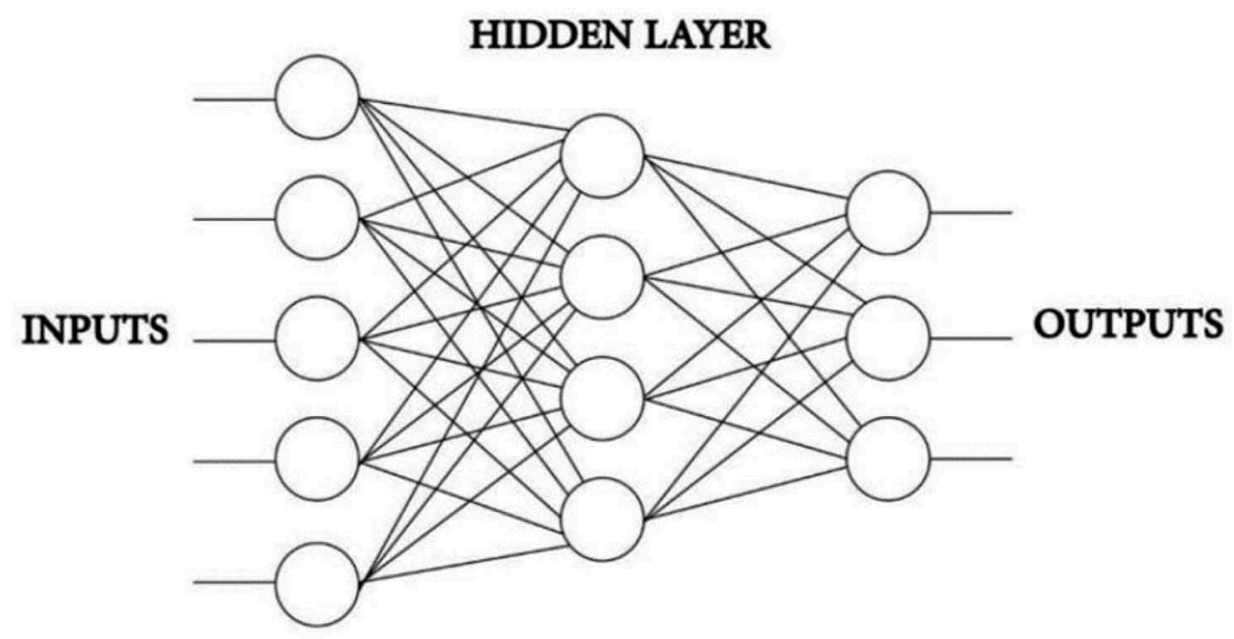

Figure 2. The basic structure of an ANN.

ANNs are programmed or trained in a way that a specific input has a particular target output. Figure 3 demonstrates this situation where the network is modified based on the difference between the target and the output until the output is close enough to the target. To train a network, many input/target pairs should be used, so as to supervise the learning process (Demuth et al. 2001).

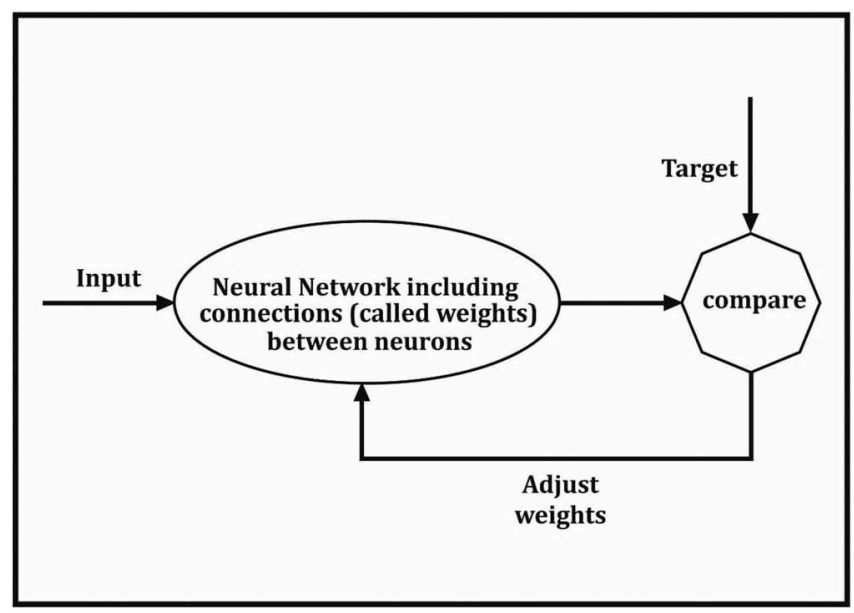

Figure 3. Basic principles of ANNs (Demuth et al. 2001).

\subsection{Multilayer Perceptron (MLP)}

Defined as one of the significant ANNs and common in engineering, the perceptron is considered as one of the most applicable networks. It is a non-recursive network which uses a supervised training algorithm. These networks are able to perform accurately a non-linear 
mapping by choosing the appropriate number of layers and neural sets. (Hagen et al. 2014, Menhaj 1998).

A MLP neural network model was chosen for the purpose of this study because it can deal with those cases, when not enough FWD deflection data is available. These networks are particularly suited to modeling complex data as provided by FWD due to the capability to learn complex nonlinear behavior.

Usually, a MLP is trained with a backpropagation (BP) algorithm (Principe et al., 2000; Rumelhart et al., 1986). The BP learning algorithm is one of the most important historical developments in neural networks. The MLP modeling with BP algorithm consists of two steps (Hagen et al. 2014, Menhaj 1998):

The route forward: in this route, the input vector is applied on the network, and its effects are transferred via hidden layers to the output layers. The created output vector in the output layer gives the real response of MLP.

The route backward: in this route, the error vectors are reverted from the last layer to the first one. In other words, in this route, the output layer, marks the beginning of the procedure, and the error vector is distributed from output to input, which means that it moves from the last layer to the first one.

The error vector is the difference between the network response (output) and the measured value (target). The error value is calculated in the second step from the output layer and distributed through the network layers throughout the network.

\subsection{Training architecture}

In this study, an MLP network is used with two hidden layers. The sigmoid function is in the first and second hidden layer and the linear function in the output layer. Further hidden layers are not considered as they complicate the problem and do not produce better results. The goal is to achieve the best result, so, certain modifications are applied to inputs, neurons, and algorithms (training functions) at each iteration. In other words, a neural network is trained for a single output with various inputs, different number of neurons and different algorithms in order to determine the best network among the possible training methods.

The input layer consists of three parameters: measuring point, thickness, and temperature. The output layer has just one parameter: deflection. The model is shown in Figure 4.

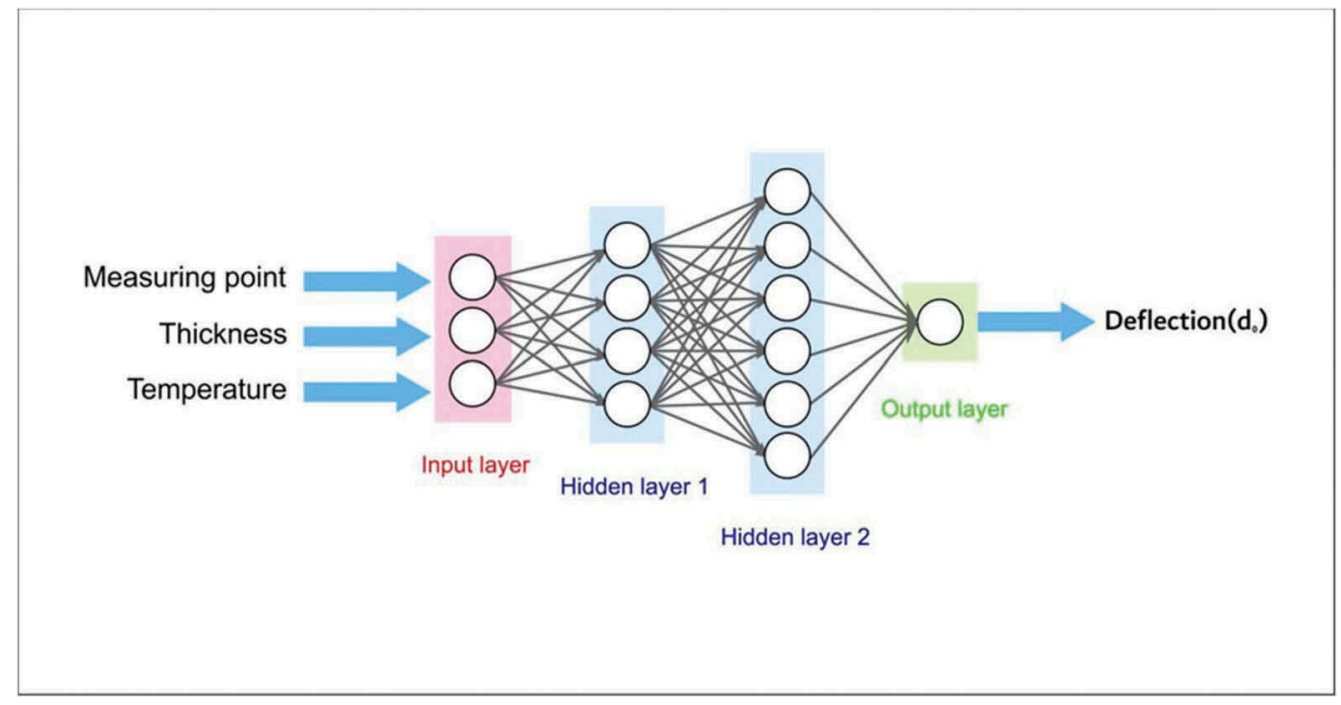

Figure 4. A schematic chart of a MLP. 


\subsection{Dividing the modeling data}

The main capability of ANN is its generalizability. It refers to the possibility of generalizing the results from previous data to new data after training. In fact, the networks are trained in a way that makes them capable of extracting trends and patterns from a specific data set and generalize them to other data. The objective is to ensure the best modeling. Data sets are divided into three sections:

1. Training data: Training data, which refers to data used during the training process, is evident, and is applied as template model. The network is trained using the bulk of data ( $70 \%$ was used in this study) to have the highest possible correspondence between input and output.

2. Validation data: A network might be trained incorrectly, as it tends to "memorize" the data and thus loses the ability to generalize. This is called overtraining (or overfitting). To avoid overtraining, the training must be validated during the process. Thus, another, independent set of data ( $15 \%$ in this study) is tested during the training in order to validate the training process and prevent overtraining from happening. Training will continue if the validation results are positive. Negative results are recorded as "fail". The simultaneous testing and training processes continue, until failed cases reach a certain extent, when the training is stopped. This means that further training would not produce any positive results. In other words, validation results have the ability to stop the training.

3. Testing data: After completion of the training process, testing data is used with independent targets, in order to validate the accuracy of learning. Testing the data is intended to produce the outcome of training. In other words, testing data $(15 \%$ in this study) provides the final answers to whether the network was trained successfully. Unlike validation data, these data do not have the ability to stop the training and are provided after the training as discussed later.

\section{CASE STUDY AND RESULTS}

For this study, FWD measuring stations were installed at $20 \mathrm{~m}$ intervals and thickness measuring stations (GPR) were placed at $8 \mathrm{~m}$ intervals. A part of thickness data produced by the same station is used to draw the matrices for input and output. As a result, 158 data series of input and output were obtained (Rahimi Nahoujy 2020). In this paper, the MLP network is implemented in the MATLAB ${ }^{\circ}$ programing language and computing environment, where the MLP model is trained with FWD data.

In order to obtain an optimum architecture, different hidden layers, neurons and training algorithms were applied. Each architecture was trained with the same input and output dataset. The models are the outcome of training measuring point, asphalt body temperature, and thickness as input and deflection (d0) as output.

This study finds that the best model has two hidden layers with 4 neurons in the first and 6 in the second hidden layer. The training algorithm used is Conjugate Gradient Backpropagation with Polak-Ribiére Update (CGP). Furthermore, R-value and performance between experimental and simulated data were determined. Figure 5 shows a diagram of performance. This network was trained for 2000 epochs and epoch No. 962 represents the best performance in this network. The network was stopped due to "Maximum epoch reached". The best performance of training, the best performance of validation and the best performance of test are $0.0031,0.0042$ and 0.0050 , respectively. In this study mean square error (MSE) was used to indicate for performance. The linear regression for training, validation, testing and the entire network is presented in Figures 6-9. 


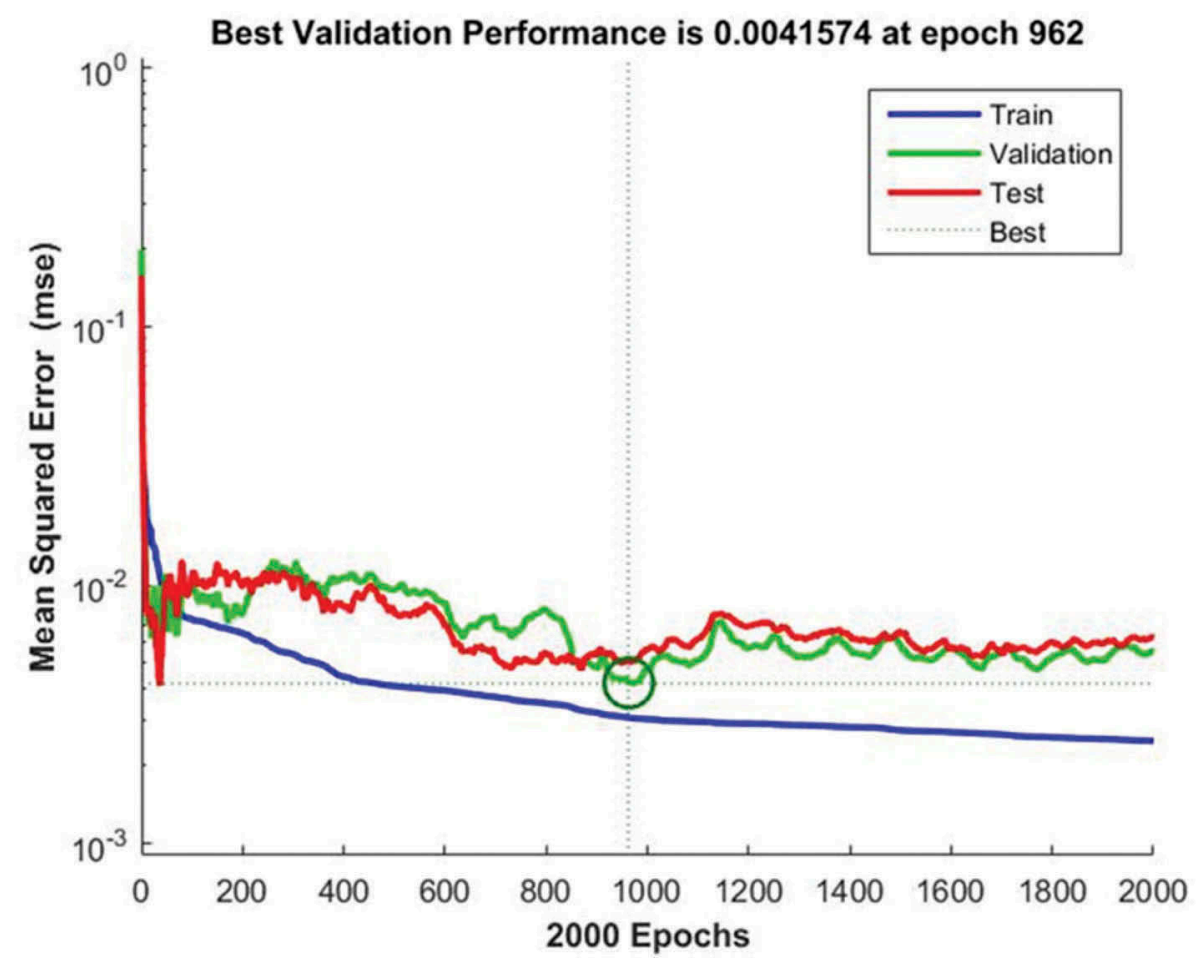

Figure 5. Performance plot for training, validation and test data sets.

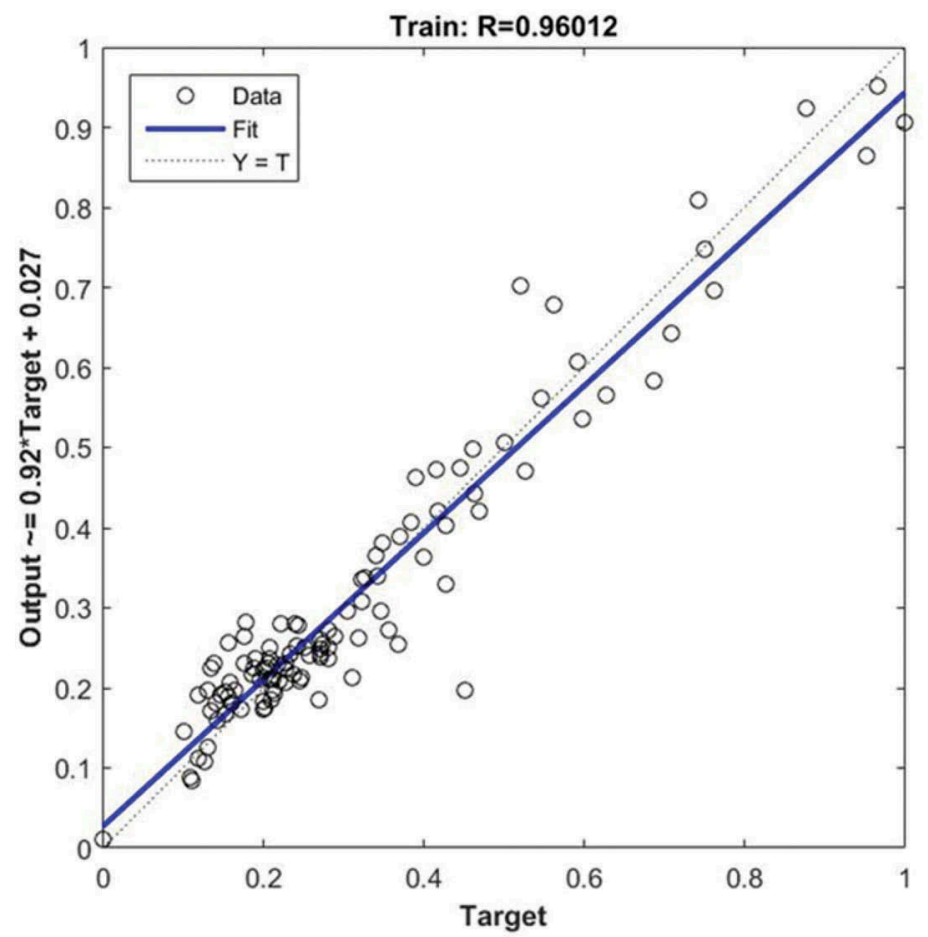

Figure 6. ANN prediction accuracy regression graphs for training data sets. 


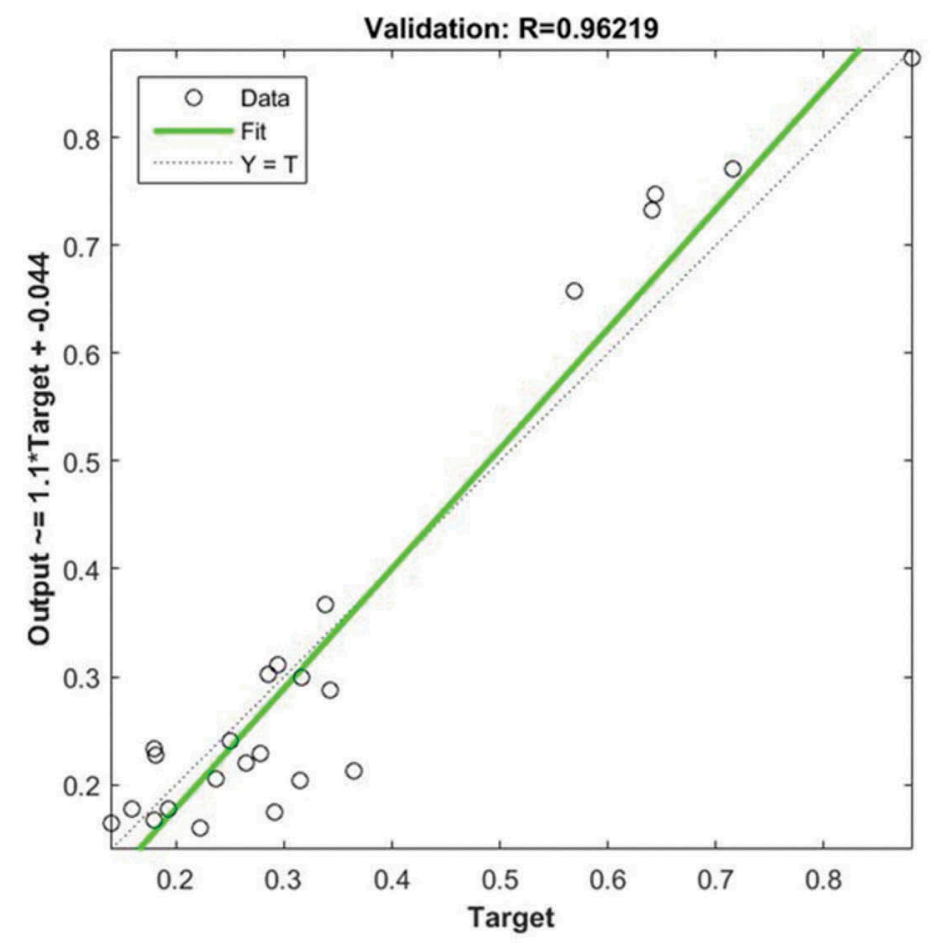

Figure 7. ANN prediction accuracy regression graphs for validation data sets.

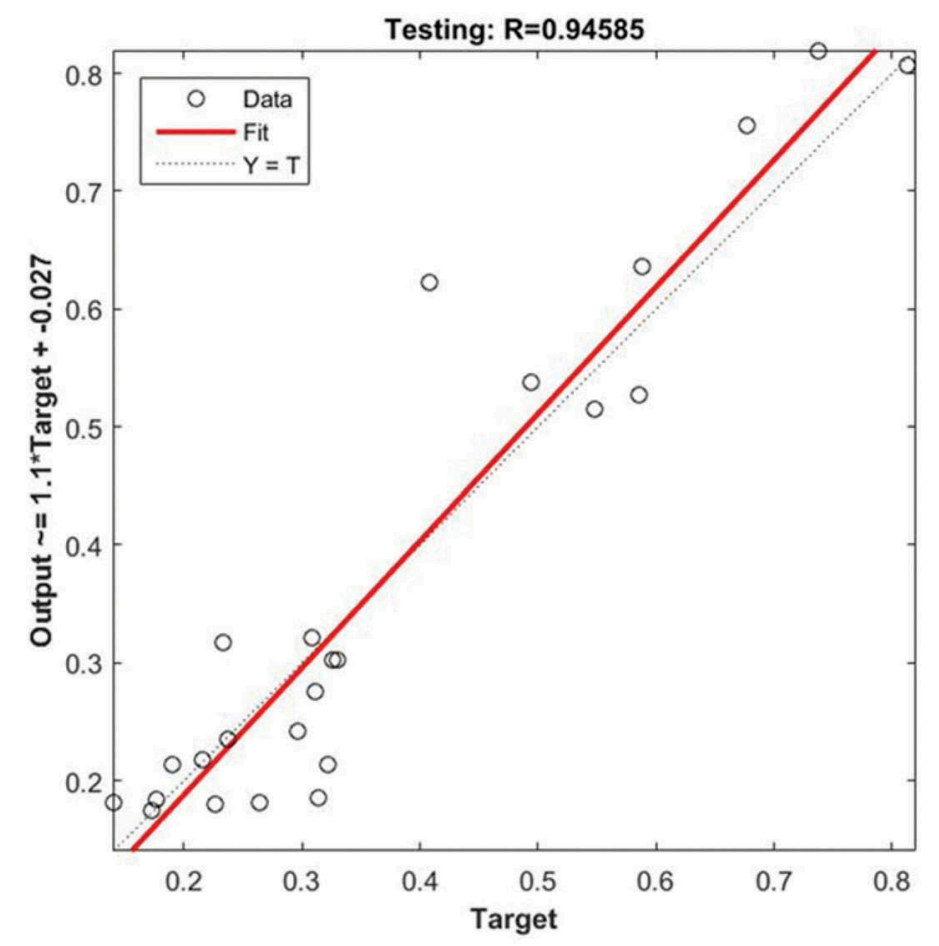

Figure 8. ANN prediction accuracy regression graphs for testing data sets. 


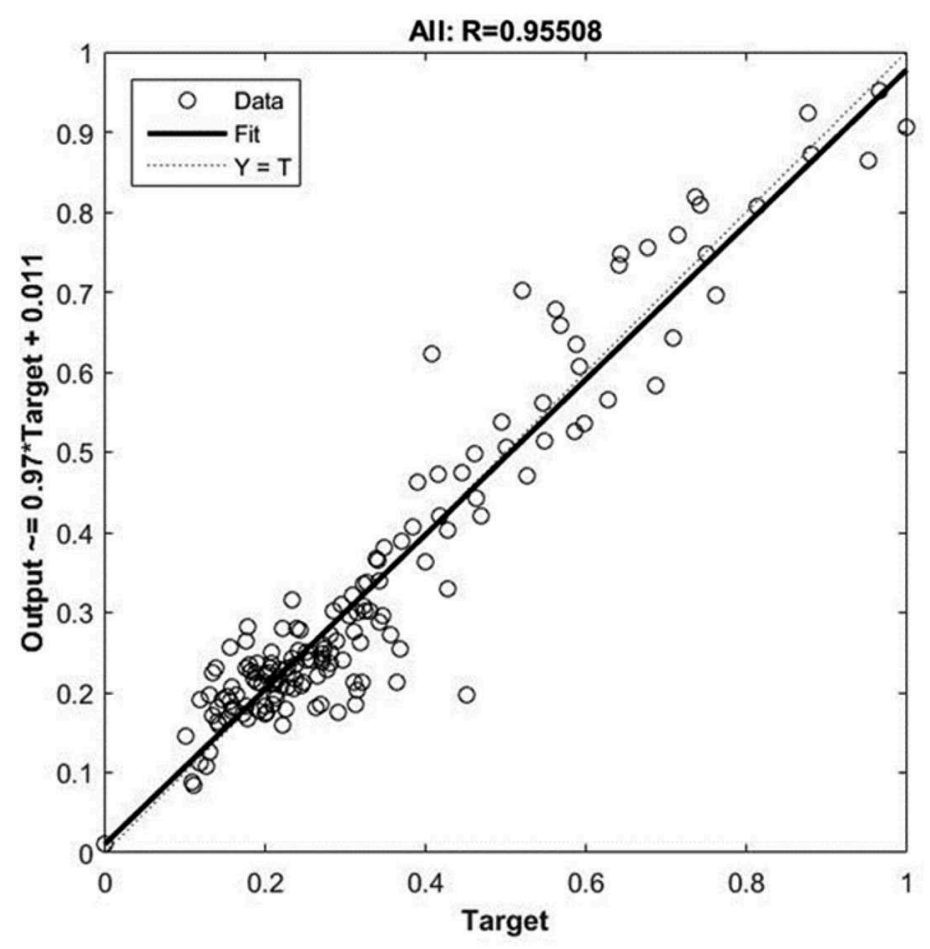

Figure 9. ANN prediction accuracy regression graphs for all data sets.

According to Figures 6-9, the R-value for training is 0.96 with a MSE of 0.0031 , indicating an excellent possibility for training the network; the R-value for validation is 0.96 with a MSE of 0.0042 . It shows that the model is robust against overtraining, the R-value for test is approx. 0.95 with a MSE of 0.0050 . Thus, it is clear that this model has very good generalization ability. The R-value for all of data is approx. 0.96. Therefore, this network is a very good network with powerful generalizability strength.

\section{CONCLUSIONS}

Deflection value is an essential index in approximating the remaining life of highway flexible pavements. It is measured by FWD in developed countries. The measurement is complicated and time consuming, so that for a short route of highway, a full day for road maintenance works is expected. Moreover, in some cases, data sets are limited, and not enough data can be obtained from FWD.

In this study, based on MLP networks, a model of ANN was developed for calculating deflection basins in flexible pavements. The basis of ANN in any engineering problem solution is to train the input and output they receive. One particular application of ANN in pavement engineering - thanks to its ability for learning complex non-linear behavior - can be investigating the complex deflection basin form to determine the structural capacity of flexible pavements.

In case of insufficient data, a perceptron can be an efficient tool for various sections of surface pavements. A perceptron can accurately do an antilinear mapping by selecting the appropriate number of layers and neural cells. MLP are among the most frequently used and most powerful neural networks when it comes to function approximation problems.

In this study, a feed-forward ANN model was developed for a route. It is based on backpropagation (BP) by a MLP for asphalt pavement. This model was trained with the available data sets, generalizing to other points where data sets were missing. 
This study showed that the neural network is able to model the FWD deflections and is characterized by powerful generalizability. After being modeled, the network can provide the hitherto unavailable data for all points between the measuring points with scant error. The best model had a MSE of $0.5 \%$ and a R-value of approx. $95 \%$ for the test data.

This method can be used to model many engineering problems caused by a lack of data. It is of high significance for highway agencies in assessing the structural capacity of flexible pavement. Fewer measuring points are necessary because the missing data can be calculated from the equation. As a consequence, this method offers a great potential for the optimization of the traditional measurements: First with regard to measurement costs, and second, it significantly enhances the accuracy of road maintenance planning, solving the problem of limited or missing data sets.

Furthermore, ANN models are a reliable computational tool to solve different complex problems in this field and could also be applied to the prediction of pavement layer moduli. For future research, it might be worth investigating, if ANN-based backcalculation models can be a solution for the analysis of the large number of pavement deflections needed for routine pavement evaluations.

\section{REFERENCES}

Attoh-Okine, N. O., 1999. Analysis of learning rate and momentum term in backpropagation neural network algorithm trained to predict pavement performance. Advances in Engineering Software. 30:291-302.

Ceylan, H., Gopalakrishnan, K., Guclu, A. 2007. Advanced approaches to characterizing nonlinear pavement system responses. Transportation Research Record, 86-94.

Ceylan, H., Guclu, A., Tutumluer, E., Thompson, M. R. (2005). Backcalculation of full-depth asphalt pavement layer moduli considering nonlinear stress-dependent subgrade behavior. International Journal of Pavement Engineering, 6(3),171-182.

Demuth, H., Beale, M., 2001. Neural network toolbox, user guide (version4). The MathWorks, Inc.

FGSV, 2008. Arbeitspapier Tragfähigkeit von Verkehrsflächenbefestigungen Teil B 2.1.Falling Weight Deflectometer (FWD): Gerätebeschreibung, Messdurchführung -Asphaltbauweisen. FGSV-Verl. Köln, 2008, FGSV 433 B 2.1

Hagan, M. T.; Demuth, H. B.; Beale, M. H.; De Jesus, O., 2014. Neural network design. Wrocław. Amazon Fulfillment Poland Sp. z o.o.

Huang, Y. H., 2004. Pavement design and analysis. Upper Saddle River, NJ. Pearson/Prentice Hall.

Jansen, D., 2009. Temperaturkorrektur von mit dem Falling-Weight-Deflectometer gemessenen Deflexionen auf Asphaltbefestigungen. Dissertation, Institute für Straßenbau und Verkehrswesen, Universität Duisburg-Essen, Schriften-reihe Heft 2, Essen, Germany.

Kim, Y., Kim, Y. R. 1998. Prediction of layer moduli from falling weight deflectometer and surface wave measurements using artificial neural network. Transportation Research Record, 1639, 53-61.

Li, M. Y., Wang, H. 2019. Development of ANN-GA program for backcalculation of pavement moduli under FWD loading with viscoelastic and nonlinear parameters. International Journal of Pavement Engineering, 20(4),490-498.

Menhaj, M. B., 1998. Fundamentals of neural networks. Computational intelligence. No. 1.

Nejad, F. M., Zakeri, H., 2012. The hybrid method and its application to smart pavement management. Metaheuristics Water Geotech Transp Eng, $439 \mathrm{H}$.

Principe, J. C., Euliano, N. R., Lefebvre, W. C., 2000. Neural and adaptive systems: fundamentals through simulations (Vol. 672). New York: Wiley.

Rahimi Nahoujy, M. 2020. An Artificial Neural Network approach to model and predict asphalt deflections as a complement to experimental measurements by Falling Weight Deflectometer. Doctoral dissertation, Ruhr-Universität Bochum, Germany.

Rumelhart, D. E.; Hinton, G. E.; Williams, R. J., 1986. Learning internal representations by error propagation. In: Parallel distributed processing: explorations in the microstructure of cognition, vol. 1, pp. 318-362. MIT Press.

Saltan, M., Terzi, S. 2008. Modeling deflection basin using artificial neural networks with cross-validation technique in backcalculating flexible pavement layer moduli. Advances in Engineering Software, 39 (7), 588-592.

Wang, H., Xie, P., Ji, R., Gagnon, J. 2020. Prediction of airfield pavement responses from surface deflections: comparison between the traditional backcalculation approach and the ANN model. Road Materials and Pavement Design, 1-16. 